\title{
ÉDITORIAL
}

\section{Commentaires de nos lecteurs : ce qu'ils nous ont dit}

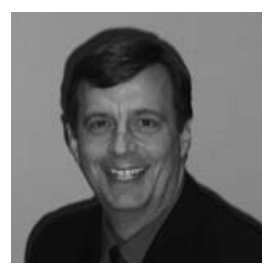

James Ducharme, MD

ENGLISH VERSION ON PAGE 6

À l'été 2007, le nouvel exécutif du conseil de rédaction du JCMU estimait qu'il était essentiel d'obtenir les commentaires des rédacteurs responsables des décisions et des rédacteurs de section et encore plus de vous, nos lecteurs. À la lumière des réponses reçues, nous allions amorcer la planification des changements éventuels au JCMU. Nous avions certes une idée des changements à apporter, comme l'indiquent les questions dirigées que nous avons posées, mais nous espérions recueillir de nombreux commentaires en posant la question ouverte suivante : quels changements aimeriez-vous voir au $J C M U$ ?

Les sondages antérieurs réalisés par l'ACMU avaient révélé que le $J C M U$ est une raison importante pour laquelle bon nombre de médecins sont membres de l'ACMU. Nous étions donc ravis de la forte participation au sondage (296 répondants), mais ce résultat ne nous a pas surpris. Voici ce que vos réponses nous ont appris :

1. Contenu canadien. Vous avez manifesté un fort désir pour que le JCMU continue de mettre l'accent sur la médecine d'urgence au Canada. Plusieurs d'entre vous étaient préoccupés par le fait que l'indexation allait faire en sorte que le $J C M U$ deviendrait simplement un autre « véhicule pour la publication ». Certains ont suggéré que le contenu d'articles provenant d'autres pays publiés dans le journal devrait pour le moins être pertinent pour les médecins du Canada. Un des thèmes qui est revenu souvent dans les commentaires était d'inclure dans le journal des nouvelles d'actualité et des discussions sur des individus et des événements au sein de la communauté de médecine d'urgence au Canada. Il nous semble que la publication d'un bulletin de l'ACMU, à distribuer possiblement en même temps que le $J C M U$, serait la meilleure façon de répondre à cette suggestion. Nous avons donc transmis cette idée au siège social de l'ACMU.

2. Version électronique. Vous étiez moins nombreux qu'on s'y attendait à avoir manifesté un intérêt à recevoir une version électronique du journal, en format PDF, par courriel ou sur votre baladeur. La plupart des répondants semblaient satisfaits du format actuel du journal. Toutefois, vu un certain intérêt pour la diffusion électronique, nous allons nous pencher sur la question. Nous en suivrons l'évolution à mesure que la génération «iPod » remplacera la génération «papier».

3. Marque du journal. Plusieurs répondants nous ont dit que la page couverture et le style étaient en quelque sorte défraîchis et nous partageons leur opinion. L'ACMU et le JCMU étudient présentement la marque du journal et, dans le cadre de cette étude, ils envisagent diverses options de rajeunissement, tant pour le journal que pour le site Web.

4. Éditoriaux. Les répondants ont exprimé un intérêt marqué pour la publication régulière d'éditoriaux traitant de sujets controversés en médecine d'urgence. Vous avez sans doute remarqué que depuis l'été dernier, nous publions de tels articles dans tous les numéros. Nous espérons pouvoir en faire autant à l'avenir. Bien que certains d'entre vous estiment que les éditoriaux ne devraient pas être à caractère politique, nous considérons qu'il est presque impossible de séparer la médecine de la politique, compte tenu du fait qu'il s'agit d'une publication médicale. Cependant, outre les thèmes à caractère politique, nous tenterons de publier des éditoriaux qui refléteront les controverses en matière de traitements ou des commentaires sur certains articles que nous publions. 
5. Méthodologie. Les commentaires étaient presque unanimes : vous voulez voir des résumés d'une page sur la méthodologie relative aux articles publiés dans le $J C M U$. Le conseil de rédaction tentera de publier trois résumés de méthodologie par an, articles qui traiteront de la méthodologie utilisée dans un article publié dans le même numéro.

6. Domaines d'intérêt. Le conseil consultatif de rédaction s'entend pour dire que l'enseignement médical constitue un pilier de la médecine d'urgence en milieu universitaire et que nous devrions publier davantage d'articles dans ce domaine. Vous avez été nombreux à nous dire que vous aimeriez lire des articles portant sur des questions médico-juridiques ainsi que des revues générales sur des sujets précis. Le JCMU a toujours encouragé les auteurs à soumettre des revues systématiques, les études narratives étant souvent moins objectives. Nous continuerons de promouvoir la soumission de tels articles dans ces domaines, quoique nous sachions fort bien que nous sommes à la merci de la volonté des auteurs de les rédiger.

Ayant reçu une si forte réponse des lecteurs du JCMU, nous exhortons maintenant les directeurs de programmes de résidence et les chefs de file en milieu universitaire de partout au pays à nous faire part de leurs commentaires et à participer encore plus activement au JCMU, dans l'espoir que ce journal sera leur premier choix de publication.

Nous avons certes entendu haut et fort votre désir de changement et de croissance, mais nous avons aussi entendu un message tout aussi fort qui exprimait la satisfaction de la plupart d'entre vous à l'égard du JCMU, tel qu'il est. Nous nous engagerons donc avec précaution sur la voie des changements, car nous aussi aimons ce journal tel quel. Nous n'avons nullement l'intention de défaire ce que Grant Innes et tant d'autres ont travaillé si fort pour créer. Nous continuerons de vous consulter au fur et à mesure que nous évoluerons, car il va sans dire que le JCMU est pour les médecins d'urgence du Canada et non pour ses rédacteurs.

Nous vous remercions d'avoir participé à ce sondage en si grand nombre. Nous espérons que ce seront toujours les lecteurs qui guideront la direction du JCMU et que ses rédacteurs ne feront que leur faciliter la voie.

Correspondance à : cjem @ caep.ca 